\title{
Analytic Form of the Full Two-Loop Five-Gluon All-Plus Helicity Amplitude
}

\author{
S. Badger, ${ }^{1}$ D. Chicherin, ${ }^{2}$ T. Gehrmann, ${ }^{3}$ G. Heinrich, ${ }^{2}$ J. M. Henn, ${ }^{2}$ T. Peraro, ${ }^{3}$ \\ P. Wasser, ${ }^{4}$ Y. Zhang, ${ }^{2,5}$ and S. Zoia ${ }^{2}$ \\ ${ }_{1}^{1}$ Institute for Particle Physics Phenomenology, Durham University, Durham DH1 3LE, United Kingdom \\ ${ }^{2}$ Max-Planck-Institut für Physik, Werner-Heisenberg-Institut, D-80805 München, Germany \\ ${ }^{3}$ Physik-Institut, Universität Zürich, Wintherturerstrasse 190, CH-8057 Zürich, Switzerland \\ ${ }^{4}$ PRISMA+Cluster of Excellence, Johannes Gutenberg University, D-55099 Mainz, Germany \\ ${ }^{5}$ Interdisciplinary Center for Theoretical Study, University of Science and Technology of China, Hefei, Anhui 230026, China
}

(Received 17 May 2019; published 13 August 2019)

\begin{abstract}
We compute the full-color two-loop five-gluon amplitude for the all-plus helicity configuration. In order to achieve this, we calculate the required master integrals for all permutations of the external legs, in the physical scattering region. We verify the expected divergence structure of the amplitude and extract the finite hard function. We further validate our result by checking the factorization properties in the collinear limit. Our result is fully analytic and valid in the physical scattering region. We express it in a compact form containing logarithms, dilogarithms, and rational functions.
\end{abstract}

DOI: 10.1103/PhysRevLett.123.071601

Introduction.-The abundant amount of data to be collected by the ATLAS and CMS Collaborations in future runs of the Large Hadron Collider at CERN opens up a new era of precision physics. Some of the most prominent precision observables are related to three-jet production $[1,2]$, which allows in-depth studies of the strong interaction up to the highest energy scales, including precision measurements of the QCD coupling constant $\alpha_{s}$ and its scale evolution. The physics exploitation of these precision data requires highly accurate theory predictions, which are obtained through the computation of higher orders in perturbation theory. Second-order corrections (next-tonext-to-leading order, NNLO) were computed recently for many two-to-two scattering processes, including twojet production [3]. A comparable level of theoretical accuracy could not, up to now, be obtained for genuine two-to-three processes, especially since the relevant matrix elements for processes involving five external partons including full color are known only up to one loop [4-6].

The evaluation of these two-loop five-parton matrix elements faces two types of challenges: to relate the large number of two-loop integrands to a smaller number of master integrals, and to compute these master integrals (two-loop five-point functions). Important progress was made most recently on both issues, with the development and application of efficient integral reduction techniques,

Published by the American Physical Society under the terms of the Creative Commons Attribution 4.0 International license. Further distribution of this work must maintain attribution to the author(s) and the published article's title, journal citation, and DOI. Funded by SCOAP. either analytical [7-14] or seminumerical [15,16], as well as with the computation of the two-loop five-point functions for planar [17-19] and nonplanar [20-22] integral topologies. The latter developments have already led to first results for two-loop five-point amplitudes in supersymmetric Yang-Mills theory [21,23] and supergravity [24,25].

Recent progress has enabled the computation of the full set of the leading-color two-loop corrections to the fiveparton amplitudes, represented in a seminumerical form [10,26-28]. These results establish the technical methodology; their evaluation is, however, too inefficient for practical use in the computation of collider cross sections. Towards this aim, analytic results are preferable, which have been obtained so far only at leading color for the fiveparton amplitudes [17,29-32]. Besides the more efficient numerical evaluation, these results also allow for detailed investigations of the limiting behavior in kinematical limits, thereby elucidating the analytic properties of scattering in QCD.

The leading-color corrections consist only of planar Feynman diagrams. At subleading color level, nonplanar diagrams and integrals contribute as well, leading to a considerable increase in complexity, both in the reduction of the integrand and in the evaluation of the master integrals. In this Letter, we make the first step towards the fully analytic evaluation of two-loop five-point amplitudes by exploiting the recently derived nonplanar two-loop five-point master integrals [20-22] to obtain an analytic expression for the two-loop five-gluon amplitude with allplus helicities [33].

Kinematics.-We study the scattering of five gluons in the all-plus helicity configuration. The corresponding amplitude has a complete permutation symmetry under the 
exchange of external gluons. The five lightlike momenta $p_{i}$ are subject to on-shell and momentum conservation conditions, $p_{i}^{2}=0$ and $\sum_{i=1}^{5} p_{i}=0$, respectively. They give rise to the following independent parity-even Lorentz invariants:

$$
X=\left\{s_{12}, s_{23}, s_{34}, s_{45}, s_{15}\right\},
$$

with $s_{i j}=2 p_{i} \cdot p_{j}$, as well as to the parity-odd invariant $\epsilon_{5}=\operatorname{tr}\left(\gamma_{5} \not \not 1_{1} \not \not_{2} \not \not_{3} \not \not_{4}\right)$. The latter is related to the Gram determinant $\Delta=\operatorname{det}\left(\left.s_{i j}\right|_{i, j=1} ^{4}\right)$ through $\epsilon_{5}^{2}=\Delta$.

Without loss of generality, we take the kinematics to lie in the $s_{12}$ scattering region. The latter is defined by all $s$-channel invariants being positive, i.e.,

$$
s_{12}>0, \quad s_{34}>0, \quad s_{35}>0, \quad s_{45}>0,
$$

and $t$-channel ones being negative, i.e.,

$$
s_{1 j}<0, \quad s_{2 j}<0, \quad \text { for } j=3,4,5,
$$

as well as by the requirement that the particle momenta are real, which implies $\Delta<0$.

The external momenta $p_{i}$ lie in four-dimensional Minkowski space. We encounter $D$-dimensional Feynman integrals, with $D=4-2 \epsilon$, and the loop momenta therefore live in $D$ dimensions. We keep the explicit dependence on the spin dimension $D_{s}=g_{\mu}^{\mu}$ of the gluon, which enters the calculation via the integrand numerator algebra. Results in the t'Hooft-Veltman [34] and four-dimensional-helicity [35] schemes can be obtained by setting $D_{s}=4-2 \epsilon$ and $D_{s}=4$, respectively. We denote $\kappa=\left(D_{s}-2\right) / 6$.

Decomposition of the amplitude in terms of color structures.-We expand the unrenormalized amplitude in the coupling $a=g^{2} e^{-\epsilon \gamma_{\mathrm{E}}} /(4 \pi)^{2-\epsilon}$ as

$$
\mathcal{A}_{5}=i g^{3} \sum_{\ell \geq 0} a^{\ell} \mathcal{A}_{5}^{(\ell)} .
$$

Because of the particular helicity configuration, the amplitude vanishes at tree level $[36,37]$ and is hence finite at one loop.

The amplitude is a vector in color space. Adopting the conventions of Ref. [38], we decompose the one- and twoloop amplitudes as

$\mathcal{A}_{5}^{(1)}=\sum_{\lambda=1}^{12} N_{c} A_{\lambda}^{(1,0)} \mathcal{T}_{\lambda}+\sum_{\lambda=13}^{22} A_{\lambda}^{(1,1)} \mathcal{T}_{\lambda}$,

$\mathcal{A}_{5}^{(2)}=\sum_{\lambda=1}^{12}\left(N_{c}^{2} A_{\lambda}^{(2,0)}+A_{\lambda}^{(2,2)}\right) \mathcal{T}_{\lambda}+\sum_{\lambda=13}^{22} N_{c} A_{\lambda}^{(2,1)} \mathcal{T}_{\lambda}$.

Here, the $\left\{\mathcal{T}_{\lambda}\right\}$ consist of 12 single traces, $\lambda=1, \ldots, 12$, and 10 double traces, $\lambda=13, \ldots, 22$. We have

$$
\begin{aligned}
\mathcal{T}_{1} & =\operatorname{Tr}(12345)-\operatorname{Tr}(15432), \\
\mathcal{T}_{13} & =\operatorname{Tr}(12)[\operatorname{Tr}(345)-\operatorname{Tr}(543)],
\end{aligned}
$$

where $\operatorname{Tr}\left(i_{1} i_{2} \ldots i_{n}\right) \equiv \operatorname{Tr}\left(T^{a_{i_{1}}} \ldots T^{a_{i_{n}}}\right)$ denotes the trace of the generators $T^{a_{i}}$ of the fundamental representation of $S U\left(N_{c}\right)$. The remaining color basis elements $\mathcal{T}_{\lambda}$ are given by permutations of $\mathcal{T}_{1}$ and $\mathcal{T}_{13}$. For the explicit expressions, see Eqs. (2.1) and (2.2) of Ref. [38].

The one-loop expression can be found in Ref. [4]. Here, we write it in a new form,

$$
A_{1}^{(1,0)}=\frac{\kappa}{5} \sum_{S_{\tau_{1}}}\left(\frac{[24]^{2}}{\langle 13\rangle\langle 35\rangle\langle 51\rangle}+2 \frac{[23]^{2}}{\langle 14\rangle\langle 45\rangle\langle 51\rangle}\right),
$$

up to $\mathcal{O}(\epsilon)$ terms. The sum runs over the subset $S_{\mathcal{T}_{\lambda}}$ of permutations of the external legs that leave $\mathcal{T}_{\lambda}$ invariant. All other terms in Eq. (5) follow from symmetry and from $U(1)$ decoupling relations.

The new representation (8) makes a symmetry property manifest. The basic rational object is invariant under conformal transformations, which are defined as [39]

$$
k_{\alpha \dot{\alpha}}=\sum_{i=1}^{5} \frac{\partial^{2}}{\partial \lambda_{i}^{\alpha} \partial \tilde{\lambda}_{i}^{\dot{\alpha}}} .
$$

The property $k_{\alpha \dot{\alpha}} \mathcal{A}_{5}^{(1)}=\mathcal{O}(\epsilon)$ is obvious term by term due to the form of the operator in Eq. (9).

In this Letter, we compute the full two-loop amplitude. The leading color single trace terms $A_{\lambda}^{(2,0)}$ were computed in Refs. [17,29]. Generalizations of the $U(1)$ decoupling relation imply that the most subleading color terms $A_{\lambda}^{(2,2)}$ can be obtained from the leading single trace $A_{\lambda}^{(2,0)}$ and the double trace terms $A_{\lambda}^{(2,1)}$ [38]. We present explicitly the result for the finite part of the double trace term $A_{13}^{(2,1)}$. The other double trace terms can be obtained by analytic continuation, as explained below.

Factorization and exponentiation of infrared divergences.-Infrared divergences (soft and collinear) in loop amplitudes factorize similarly to ultraviolet divergences, in the following way:

$$
\mathcal{A}=\mathcal{Z} \mathcal{A}^{f} .
$$

Here, the factor $\mathcal{Z}$ is a matrix in color space. It contains all infrared divergences, in the sense that we can define an infrared finite hard function according to

$$
\mathcal{H}=\lim _{\epsilon \rightarrow 0} \mathcal{A}^{f} .
$$

For massless scattering amplitudes, $\mathcal{Z}$ is known to three loops; see Refs. [40-43]. In the present case, the tree-level amplitude vanishes, and we therefore need only the one-loop part of the infrared matrix, $\mathcal{Z}=\mathbf{1}+a \mathcal{Z}^{(1)}$, with 

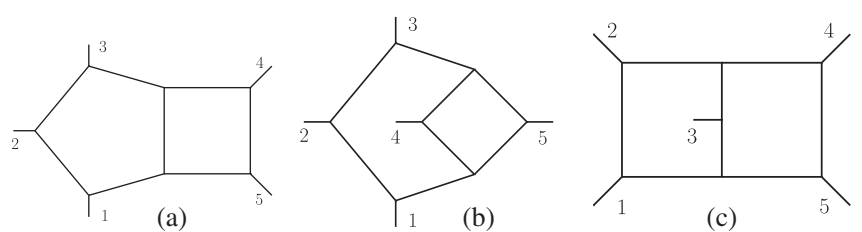

(c)

FIG. 1. Two-loop five-particle Feynman integral topologies.

$$
\mathcal{Z}^{(1)}=-\frac{e^{\epsilon \gamma_{\mathrm{E}}}}{\epsilon^{2} \Gamma(1-\epsilon)} \sum_{i \neq j}^{5} \overrightarrow{\mathbf{T}}_{i} \cdot \overrightarrow{\mathbf{T}}_{j}\left(\frac{\mu^{2}}{-s_{i j}}\right)^{\epsilon},
$$

where $\mu$ is the dimensional regularization scale, and $\overrightarrow{\mathbf{T}}_{i}=\left\{\mathbf{T}_{i}^{a}\right\}$ are the generators of $S U\left(N_{c}\right)$ in the adjoint representation of gluon $i$, which act according to $\mathbf{T}_{i}^{b} T^{a_{i}}=$ $-i f^{b a_{i} c_{i}} T^{c_{i}}$. We set $\mu=1$. The explicit dependence can be recovered from dimensional analysis.

Two-loop integrand and finite field reduction to uniform-weight master integrals.-The starting point of our calculation is the integrand presented in Ref. [33]. The latter was obtained using modern generalized unitarity techniques. It is given in terms of the integrals of the type shown in Fig. 1 (and similar integrals corresponding to subgraphs), with certain numerator factors. We begin by rewriting scalar products of $-2 \epsilon$-dimensional components of loop momenta in terms of Gram determinants; see, e.g., Ref. [22]. In this way, one obtains numerators with up to degree five for the eight-propagator integrals shown in Fig. 1, as well as some of degree six for the one-loop squared sectors. This is significantly higher as compared to the previous calculations in $\mathcal{N}=4$ super Yang-Mills theory [21,23] and $\mathcal{N}=8$ supergravity [24,25], where numerators of up to degree one and two were required, respectively.

We set up a system of integration by parts (IBP) identities with the help of LiTERED [44]. The task is to reduce the IBP system to a minimal required set of reduction identities. This is a difficult problem, and to solve it we profit from novel finite field and functional reconstruction techniques [7,9]. To do this, we solve the system, modulo prime integers, for numerical (rational) values of $\epsilon$ and of the kinematic invariants $s_{i j}$, using a custom linear solver for sparse systems. In doing so, we use a basis of dlog [22,45-47] master integrals as preferred integrals during the solution. We then reconstruct the analytic results from these numerical evaluations using a multivariate reconstruction algorithm, based on the one described in Ref. [9]. The calculation is further significantly simplified by reconstructing reduction tables only for the relevant combinations of integrals appearing in the representation of the amplitude.

Analytic results for master integrals in the $s_{12}$ scattering region.-In previous work, the planar master integrals shown in Fig. 1(a) were computed in all kinematic scattering regions [17-19]; the master integrals of
Figs. 1(b) and 1(c) were computed in one kinematic region only.

Since the integrals enter the amplitude in all different permutations of the external legs, we need to know them in several kinematic regions. In principle, the answer in different kinematic regions can be obtained via analytic continuation; see Refs. $[19,48]$. Here, we adopt a different strategy: we consider all permutations of all required master integrals, together with the differential equations they satisfy, and compute them directly in the $s_{12}$ channel. Taking the permutations of the differential equations is unambiguous, as the differential matrices are rational functions of the kinematics. In order to streamline the calculation, we also identify relations between integrals with permuted external legs. In this way, we do not need to continue the functions analytically. This workflow is also less error-prone, as all steps are completely automatic.

The dlog master integrals $\vec{f}$ of each family satisfy a differential equation of the form [46]

$$
\partial_{X} \vec{f}(X, \epsilon)=\epsilon \partial_{X}\left[\sum_{i=1}^{31} a_{i} \log W_{i}(X)\right] \vec{f}(X, \epsilon),
$$

where $a_{i}$ are constant matrices, and $W_{i}(X)$ are letters of the so-called pentagon alphabet [49], algebraic functions of the kinematic variables $X$ encoding the branch cut structure of the solution. The matrices $a_{i}$ are peculiar to the family and to the precise choice of basis $\vec{f}$, but the set of letters $\left\{W_{i}\right\}$ is the same for all massless two-loop five-particle integrals.

Solving the differential equations requires a boundary point. We choose

$$
X_{0}=\{3,-1,1,1,-1\} \text {. }
$$

This point lies in the $s_{12}$ scattering region and is symmetric under $p_{1} \leftrightarrow p_{2}$, or any permutation of $\left\{p_{3}, p_{4}, p_{5}\right\}$. We fix the boundary values analytically by requiring the absence of unphysical singularities. See Refs. [19,20,48] for a more detailed discussion. In this way, all boundary values are related to a few simple integrals. The latter are found in the literature [50,51].

We verify the boundary values numerically for two permutations of the integrals of Fig. 1(c) and for some integrals of Fig. 1(b). This is done by computing all master integrals numerically, using PYSECDEC [52,53], at the symmetric point $X_{0}$. We find it convenient to do this for an integral basis in $D=6-2 \epsilon$ dimensions. Moreover, we check the boundary values of the planar integrals of Fig. 1(a) against the program provided in Ref. [19].

We expand the solution to (13) in $\epsilon$ up to order $\epsilon^{4}$, corresponding to weight-four functions. The latter are expressed in terms of Chen iterated integrals. We adopt the same notation as in Ref. [19] and write the iterated integrals as 


$$
\begin{aligned}
{\left[W_{i_{1}}, \ldots, W_{i_{n}}\right]_{X_{0}}(X)=} & \int_{\gamma} d \log W_{i_{n}}\left(X^{\prime}\right) \\
& \times\left[W_{i_{1}}, \ldots, W_{i_{n-1}}\right]_{X_{0}}\left(X^{\prime}\right),
\end{aligned}
$$

where the integration path $\gamma$ connects the boundary point $X_{0}$ to $X$. In the following we do not explicitly show the dependence on the kinematic point $X$.

In order to have a common notation, we rewrite the $\mathcal{Z}$ factor, as well as all other ingredients to the hard function, in the same iterated integral notation. In this way, we analytically perform simplifications for the hard function at the level of iterated integrals. Remarkably, as observed previously for the planar case, we find that all weight-three and weight-four pieces cancel out. Therefore, we only need iterated integrals up to weight two. We rewrite them in terms of logarithms and dilogarithms. For example,

$$
\begin{aligned}
{\left[W_{1}\right]_{X_{0}} } & =\log \left(s_{12} / 3\right), \\
{\left[W_{5} / W_{2}, W_{12} / W_{2}\right]_{X_{0}} } & =-\mathrm{Li}_{2}\left(1-s_{15} / s_{23}\right) .
\end{aligned}
$$

Note that all functions are manifestly real valued in the $s_{12}$ channel. As a consequence, imaginary parts can only appear explicitly through the boundary values.

The analytic integrand expression, the IBP reductions, and the $\epsilon$ expansion of the master integrals in terms of iterated integrals, as well as the infrared subtraction, are combined numerically using finite fields. From this method, we analytically reconstruct the hard function.
At this stage, we make a remarkable observation: all dilogarithms and logarithms, as well as all imaginary parts, can be absorbed into (the finite part of) one-mass box functions, which are defined as

$$
\begin{aligned}
I_{123 ; 45}= & \operatorname{Li}_{2}\left(1-s_{12} / s_{45}\right)+\operatorname{Li}_{2}\left(1-s_{23} / s_{45}\right) \\
& +\log ^{2}\left(s_{12} / s_{23}\right)+\pi^{2} / 6
\end{aligned}
$$

Considering all permutations of external momenta provides 30 independent functions. The analytic continuation of the latter to the physical scattering region is simply achieved by adding a small positive imaginary part to each two-particle Mandelstam invariant, $s_{i j} \rightarrow s_{i j}+i 0$. This procedure correctly generates all imaginary parts in the amplitude.

Analytic result for the hard function.-We express the hard function in the same coupling expansion (4) and color decomposition (6) as the amplitude. It can be written in terms of just two color components,

$$
\mathcal{H}^{(2)}=\sum_{S_{5} / S_{\mathcal{T}_{1}}} \mathcal{T}_{1} \mathcal{H}_{1}^{(2)}+\sum_{S_{5} / S_{\mathcal{T}_{13}}} \mathcal{T}_{13} \mathcal{H}_{13}^{(2)},
$$

where each sum runs over the 5 ! permutations of the external legs, $S_{5}$, modulo the subset $S_{\mathcal{T}_{\lambda}}$ of permutations that leave $\mathcal{T}_{\lambda}$, and thus $\mathcal{H}_{\lambda}^{(2)}$, invariant.

Since the most subleading color components $\mathcal{H}_{\lambda}^{(2,2)}$ can be obtained from the planar $\mathcal{H}_{\lambda}^{(2,0)}$ and double trace $\mathcal{H}_{\lambda}^{(2,1)}$ ones through color relations [38], we present explicitly only the latter here:

$$
\begin{aligned}
\mathcal{H}_{1}^{(2,0)}= & \sum_{S_{\mathcal{T}_{1}}}\left\{-\kappa \frac{[45]^{2}}{\langle 12\rangle\langle 23\rangle\langle 31\rangle} I_{123 ; 45}+\kappa^{2} \frac{1}{\langle 12\rangle\langle 23\rangle\langle 34\rangle\langle 45\rangle\langle 51\rangle}\left[5 s_{12} s_{23}+s_{12} s_{34}+\frac{\operatorname{tr}_{+}^{2}(1245)}{s_{12} s_{45}}\right]\right\}, \\
\mathcal{H}_{13}^{(2,1)}= & \sum_{S_{\mathcal{T}_{13}}}\left\{\kappa \frac{[15]^{2}}{\langle 23\rangle\langle 34\rangle\langle 42\rangle}\left[I_{234 ; 15}+I_{243 ; 15}-I_{324 ; 15}-4 I_{345 ; 12}-4 I_{354 ; 12}-4 I_{435 ; 12}\right]\right. \\
& \left.-6 \kappa^{2}\left[\frac{s_{23} \operatorname{tr}_{-}(1345)}{s_{34}\langle 12\rangle\langle 23\rangle\langle 34\rangle\langle 45\rangle\langle 51\rangle}-\frac{3}{2} \frac{[12]^{2}}{\langle 34\rangle\langle 45\rangle\langle 53\rangle}\right]\right\},
\end{aligned}
$$

where $I$ was defined in Eq. (18), and $\operatorname{tr}_{ \pm}(i j k l):=$ $\frac{1}{2} \operatorname{tr}\left[\left(1 \pm \gamma_{5}\right) \not \not_{i} \not \check{p}_{j} \not p_{k} \not p_{l}\right]$.

The planar component (20) is in agreement with the previous result in the literature [17]. The nonplanar one (21) is entirely new. Remarkably, it exhibits the same striking simplicity: all functions of weight one, three, and four cancel out, and the remaining weight-two ones can all be expressed as permutations of the one-mass box function. While the calculation was performed in the $s_{12}$ scattering region, the above formula can be analytically continued to any other region by the $i 0$ prescription mentioned above.
Note that the rational factors multiplying the transcendental part of the hard function (20) and (21) are permutations of one object that appeared already in the one-loop amplitude (8). Remarkably, this object is conformally invariant. Moreover, the weight-two functions accompanying it are also governed by conformal symmetry. The latter manifests itself through anomalous conformal Ward identities [54-56].

Verification of correct collinear factorization.-In the limit where particles 1 and 2 are collinear, the full color five-gluon amplitude factorizes as follows: 


$$
\begin{aligned}
& \mathcal{A}^{(2)}\left(1^{+}, 2^{+}, 3^{+}, 4^{+}, 5^{+}\right) \\
& \stackrel{1 \| 2}{\rightarrow} \mathcal{A}^{(2)}\left(P^{+}, 3^{+}, 4^{+}, 5^{+}\right) \operatorname{Split}^{(0)}\left(-P^{-} ; 1^{+}, 2^{+}\right) \\
& \quad+\mathcal{A}^{(1)}\left(P^{+}, 3^{+}, 4^{+}, 5^{+}\right) \operatorname{Split}^{(1)}\left(-P^{-} ; 1^{+}, 2^{+}\right) \\
& \quad+\mathcal{A}^{(1)}\left(P^{-}, 3^{+}, 4^{+}, 5^{+}\right) \operatorname{Split}^{(1)}\left(-P^{+} ; 1^{+}, 2^{+}\right),
\end{aligned}
$$

where the sum goes over the color index of the gluon labeled by " $P$ ". After inserting expressions for the splitting amplitudes $\operatorname{Split}^{(\ell)}$ [57-60] and four-gluon amplitudes $[61,62]$, we rewrite the collinear limit in terms of the trace decomposition (6).

We verify the limits 1|| $2,2|| 3$ and 3|| 4 of the double trace term $\mathcal{T}_{13}$. It vanishes in the first two limits but has a nontrivial structure in the $3 \| 4$ limit. We find perfect agreement.

Discussion and outlook.-In this Letter, we analytically computed, for the first time, all integrals needed for twoloop massless five-particle scattering amplitudes in the physical scattering region. This required computing the master integrals in all permutations of external legs, including their boundary values, in the physical scattering region.

In view of future phenomenological applications, it is highly desirable to provide fast numerical implementations of the nonplanar pentagon functions computed here, for example, along the lines of [19].

We used the expressions for the master integrals to analytically compute the five-gluon all-plus helicity amplitude at two loops. The amplitude has the correct singularity structure and collinear behavior. In the infrared-subtracted finite part, we observed remarkable cancellations of all weight-one, three, and four functions.

Intriguingly, we found that parts of the amplitude are governed by conformal symmetry. It would be interesting to find an explanation for these observations.

Our work opens the door for further analytic calculations of massless two-loop five-particle amplitudes. On the one hand, the complete information on the integral functions is now available. On the other hand, the integral reductions required for the present calculation are of comparable complexity as to what is expected to be needed for other helicity amplitudes, or amplitudes including fermions.

We are grateful to T. Ahmed and B. Mistlberger for useful correspondence, and to S. Jahn for useful discussions. We thank the HPC groups at JGU Mainz and MPCDF for support. S. B. is supported by an STFC Rutherford Grant No. ST/L004925/1. This research received funding from the Swiss National Science Foundation (Ambizione Grant No. PZ00P2 161341), the European Research Council (ERC) under the European Union's Horizon 2020 Research and Innovation Programme, Novel structures in scattering amplitudes (Grant Agreement No. 725110), and High precision multi-jet dynamics at the LHC (Grant Agreement
No. 772009), under the Marie Skłodowska-Curie Grant Agreement No. 746223, and from the COST Action CA16201 Particleface.

[1] M. Aaboud et al. (ATLAS Collaboration), Eur. Phys. J. C 77, 872 (2017).

[2] A. M. Sirunyan et al. (CMS Collaboration), J. High Energy Phys. 12 (2018) 117.

[3] J. Currie, A. Gehrmann-De Ridder, T. Gehrmann, E. W. N. Glover, A. Huss, and J. Pires, Phys. Rev. Lett. 119, 152001 (2017).

[4] Z. Bern, L. J. Dixon, and D. A. Kosower, Phys. Rev. Lett. 70, 2677 (1993).

[5] Z. Bern, L. J. Dixon, and D. A. Kosower, Nucl. Phys. B437, 259 (1995).

[6] Z. Kunszt, A. Signer, and Z. Trocsanyi, Phys. Lett. B 336, 529 (1994).

[7] A. von Manteuffel and R. M. Schabinger, Phys. Lett. B 744, 101 (2015).

[8] K. J. Larsen and Y. Zhang, Phys. Rev. D 93, 041701(R) (2016).

[9] T. Peraro, J. High Energy Phys. 12 (2016) 030.

[10] S. Badger, C. Brønnum-Hansen, H. B. Hartanto, and T. Peraro, Phys. Rev. Lett. 120, 092001 (2018).

[11] J. Boehm, A. Georgoudis, K. J. Larsen, M. Schulze, and Y. Zhang, Phys. Rev. D 98, 025023 (2018).

[12] J. Boehm, A. Georgoudis, K. J. Larsen, H. Schönemann, and Y. Zhang, J. High Energy Phys. 09 (2018) 024.

[13] D. A. Kosower, Phys. Rev. D 98, 025008 (2018).

[14] H. A. Chawdhry, M. A. Lim, and A. Mitov, Phys. Rev. D 99, 076011 (2019).

[15] H. Ita, Phys. Rev. D 94, 116015 (2016).

[16] S. Abreu, F. Febres Cordero, H. Ita, M. Jaquier, and B. Page, Phys. Rev. D 95, 096011 (2017).

[17] T. Gehrmann, J. M. Henn, and N. A. Lo Presti, Phys. Rev. Lett. 116, 062001 (2016); 116, 189903 (2016).

[18] C. G. Papadopoulos, D. Tommasini, and C. Wever, J. High Energy Phys. 04 (2016) 078.

[19] T. Gehrmann, J. M. Henn, and N. A. Lo Presti, J. High Energy Phys. 10 (2018) 103.

[20] D. Chicherin, T. Gehrmann, J. M. Henn, N. A. Lo Presti, V. Mitev, and P. Wasser, J. High Energy Phys. 03 (2019) 042.

[21] S. Abreu, L. J. Dixon, E. Herrmann, B. Page, and M. Zeng, Phys. Rev. Lett. 122, 121603 (2019).

[22] D. Chicherin, T. Gehrmann, J. M. Henn, P. Wasser, Y. Zhang, and S. Zoia, Phys. Rev. Lett. 123, 041603 (2019).

[23] D. Chicherin, T. Gehrmann, J. M. Henn, P. Wasser, Y. Zhang, and S. Zoia, Phys. Rev. Lett. 122, 121602 (2019).

[24] D. Chicherin, T. Gehrmann, J. M. Henn, P. Wasser, Y. Zhang, and S. Zoia, J. High Energy Phys. 03 (2019) 115.

[25] S. Abreu, L. J. Dixon, E. Herrmann, B. Page, and M. Zeng, J. High Energy Phys. 03 (2019) 123.

[26] S. Badger, H. Frellesvig, and Y. Zhang, J. High Energy Phys. 12 (2013) 045.

[27] S. Abreu, F. Febres Cordero, H. Ita, B. Page, and M. Zeng, Phys. Rev. D 97, 116014 (2018).

[28] S. Abreu, F. Febres Cordero, H. Ita, B. Page, and V. Sotnikov, J. High Energy Phys. 11 (2018) 116. 
[29] D. C. Dunbar and W. B. Perkins, Phys. Rev. D 93, 085029 (2016).

[30] S. Badger, C. Brønnum-Hansen, H. B. Hartanto, and T. Peraro, J. High Energy Phys. 01 (2019) 186.

[31] S. Abreu, J. Dormans, F. F. Cordero, H. Ita, and B. Page, Phys. Rev. Lett. 122, 082002 (2019).

[32] S. Abreu, J. Dormans, F. Febres Cordero, H. Ita, B. Page, and V. Sotnikov, J. High Energy Phys. 05 (2019) 084.

[33] S. Badger, G. Mogull, A. Ochirov, and D. O'Connell, J. High Energy Phys. 10 (2015) 064.

[34] G. 't Hooft and M. J. G. Veltman, Nucl. Phys. B44, 189 (1972).

[35] Z. Bern, A. De Freitas, L. J. Dixon, and H. L. Wong, Phys. Rev. D 66, 085002 (2002).

[36] S. J. Parke and T. R. Taylor, Phys. Lett. 157B, 81 (1985); 174, 465(E) (1986).

[37] M. L. Mangano and S. J. Parke, Phys. Rep. 200, 301 (1991).

[38] A. C. Edison and S. G. Naculich, Nucl. Phys. B858, 488 (2012).

[39] E. Witten, Commun. Math. Phys. 252, 189 (2004).

[40] S. Catani, Phys. Lett. B 427, 161 (1998).

[41] G. F. Sterman and M. E. Tejeda-Yeomans, Phys. Lett. B 552, 48 (2003).

[42] L. J. Dixon, L. Magnea, and G. F. Sterman, J. High Energy Phys. 08 (2008) 022.

[43] O. Almelid, C. Duhr, and E. Gardi, Phys. Rev. Lett. 117, 172002 (2016).

[44] R. N. Lee, arXiv:1212.2685.

[45] N. Arkani-Hamed, J. L. Bourjaily, F. Cachazo, and J. Trnka, J. High Energy Phys. 06 (2012) 125.

[46] J. M. Henn, Phys. Rev. Lett. 110, 251601 (2013).
[47] J. M. Henn, T. Peraro, M. Stahlhofen, and P. Wasser, Phys. Rev. Lett. 122, 201602 (2019).

[48] J. M. Henn and B. Mistlberger, J. High Energy Phys. 05 (2019) 023.

[49] D. Chicherin, J. Henn, and V. Mitev, J. High Energy Phys. 05 (2018) 164.

[50] T. Gehrmann and E. Remiddi, Nucl. Phys. B601, 248 (2001).

[51] T. Gehrmann and E. Remiddi, Nucl. Phys. B601, 287 (2001).

[52] S. Borowka, G. Heinrich, S. Jahn, S. P. Jones, M. Kerner, J. Schlenk, and T. Zirke, Comput. Phys. Commun. 222, 313 (2018).

[53] S. Borowka, G. Heinrich, S. Jahn, S. P. Jones, M. Kerner, and J. Schlenk, Comput. Phys. Commun. 240, 120 (2019).

[54] D. Chicherin and E. Sokatchev, J. High Energy Phys. 04 (2018) 082.

[55] D. Chicherin, J. M. Henn, and E. Sokatchev, Phys. Rev. Lett. 121, 021602 (2018).

[56] S. Zoia, Proc. Sci. LL2018 (2018) 037.

[57] S. J. Parke and T. R. Taylor, Phys. Rev. Lett. 56, 2459 (1986).

[58] F. A. Berends and W. T. Giele, Nucl. Phys. B306, 759 (1988).

[59] M. L. Mangano, S. J. Parke, and Z. Xu, Nucl. Phys. B298, 653 (1988).

[60] Z. Bern, L. J. Dixon, D. C. Dunbar, and D. A. Kosower, Nucl. Phys. B425, 217 (1994).

[61] Z. Bern and D. A. Kosower, Nucl. Phys. B379, 451 (1992).

[62] Z. Bern, L. J. Dixon, and D. A. Kosower, J. High Energy Phys. 01 (2000) 027. 